\title{
Knowledge and Awareness Regarding Safe Drug Disposal System among General Population of India
}

\author{
Shwetha $\mathbf{N}^{*}$ and Ateendra Jha \\ Department of Pharmacy Practice, Srinivas College of Pharmacy, Valachil, Post Farangipete, Mangalore, Karnataka 574143, India
}

\begin{abstract}
Thousands of drugs are releasing into the market day by day in order to treat various diseases and to promote the health of growing population of India. But when they no longer needed their disposal become necessary because drug is a chemical and once it expires it will become a toxic agent. The people may have expired drugs, unused or unwanted medicines which may occur as a result of non-adherence, excessive storage of OTC medication or disuse of drugs. The USFDA initiated 'drug take back programme' to prevent the entry of these medication into environment. But in India drug take back programmer is not functional. The country is now facing the numerous problems due to improper drug disposal methods such as burning, flushing into toilet and throwing somewhere or into waste-basket which may lead to environmental contamination and pollution, contamination of water supplies and other local sources used by community and wildlife which will results in serious environmental and health hazards like toxicity, accidental poisoning and drug abuse, development of drug resistance problems and even death. Since the drug take back programme is not functional in the developing countries like India several cases of toxicity and accidental exposure are seen day by day. So an online survey was conducted in general population in order to check the knowledge of people regarding safe drug disposal system. From the data, $73 \%$ of 214 people are not aware of safe and effective disposal of drugs without causing any harmful effect to nature and public.
\end{abstract}

Keywords: Expired drugs; Improper drug disposal; Environmental hazards; Drug take back programme

\section{Introduction}

Medicines are integral part of health care system. Each and every day millions of people are taking the medicine for one or other purpose. They may have any expired drugs, unused or unwanted medicines which may occur as a result of non-adherence, excessive storage of OTC medication or disuse of drugs [1-4]

The common methods used by general population to dispose these medications are throwing into dustbin or somewhere, burning, flushing into the sink or toilet [5-7].

\section{Throwing into Dustbin}

It is the most common and improper method used by the people. When the medications are no longer required they will just throw the medications into the dustbin or store in home itself. As the medications are made attractive for better patient compliance, there is a chance of taking these expired or unused medicines by children or pets which may lead to serious toxicity or poisoning condition. Not only this, when these medicines are disposed by means of throwing into waste basket, they will end up with reaching landfills with other solid waste which will result in serious environmental and health hazards $[1,8]$.

\section{Burning}

It is another improper way of disposal of medicine. Chemically, a medicine is either organic or inorganic compound. Obviously burning of a chemical compound in the nature will result in the release of several chemical pollutants to the atmosphere. It is very dangerous to inhale the air polluted with these contents. The serious problems such as drug toxicity, drug resistance and sudden death due to poisoning condition may arise because of the burning of the medicines.

\section{Flushing into Sink or Toilet}

This method is mainly used for disposal of liquid medicines that are either unused or expired. Flushing a drug into sink or toilet will results in the contamination of water sources. A major study finds 'excessively high' levels of drug residues in water bodies of Hyderabad allowing microbes to build resistance to medicines $[9,10]$. Not only this, a new study by researchers at the Indian Institute of Technology, Delhi found high levels of $\beta$-lactamresistant bacteria and genes, particularly carbapenem and extended spectrum $\beta$-lactam (ESBL)-resistant bacteria and genes from 12 STPs in Delhi 12. These two cases indicate that the reason for treatment failure may be improper drug disposal also.

However, the chances of toxicity are comparatively less than that of other two methods because of large quantity of dilution [2]. USFDA says, it is an alternative method to dispose certain medicines (mainly centrally acting) if drug take back system is not available in your area [2].

To prevent the dangerous consequences of improper drug disposal system the FDA initiated the National drug take bake programme on $25^{\text {th }}$ September 2010 that aims to provide a safe, convenient, and responsible means of disposing of prescription drugs, while also educating the general public about the potential for abuse of medications. It helps to divert medicines from entering the environment. In past years, they have collected more than 200 pounds of unused medications that would have otherwise disposed improperly. But in India, the drug take back programme is not functional effectively which may be reason for improper drug disposal [4,5,7].

*Corresponding author: Shwetha N, Department of Pharmacy Practice, Srinivas College of Pharmacy, Valachil, Post Farangipete, Mangalore, Karnataka 574143, India, Tel: +91 8105746172; E-mail: nshwetha172@gmail.com

Received: March 23, 2018; Accepted April 07, 2018; Published April 14, 2018

Citation: Shwetha N, Jha A (2018) Knowledge and Awareness Regarding Safe Drug Disposal System among General Population of India. J Pharmacovigil 6: 256 doi:10.4172/2329-6887.1000256

Copyright: (c) 2018 Shwetha N, et al. This is an open-access article distributed under the terms of the Creative Commons Attribution License, which permits unrestricted use, distribution, and reproduction in any medium, provided the original author and source are credited. 


\section{Objective}

Improper drug disposal methods are hazardous to the nature and life which are common in developing countries where there is no significant development in the drug take back programme. So the aim of this study is to check the knowledge and to create awareness regarding safe drug disposal system among the general population of India.

\section{Experimental Methods}

An online survey was conducted with the help of questionnaire in order to collect the data from the general population of above 10 years to check their knowledge regarding drug disposal system. Articles were collected from the online sources such as PUBMED, WHO, FDA and GOOGLE SCHOLAR and questionnaires were prepared by using these articles. In this survey, 214 people were responded and the responses which have been received were assessed, analyzed and interpreted by using Microsoft Excel 2010.

\section{Results}

In the survey, 214 people are participated. Out of them $54 \%$ are females and $47 \%$ are males. Among them, $28 \%$ of people are using burning method for the disposal of unused, expired or unwanted drugs, $40.18 \%$ of people are throwing into waste basket, $19.60 \%$ of people are flushing them into sink or toilet and $9.3 \%$ of them store them in their home itself. Only $2.80 \%$ of people are returning the unused drugs to the pharmacy. The data also shows that, $14 \%$ of antibiotics, $21.1 \%$ of pain killers, $21 \%$ of prescribed medicines and $27.60 \%$ drugs are remaining with patient even after therapy. This may be because of non-adherence and other reasons. The data also indicates that only $27 \%$ of people are aware of drug take back programme and majority of the people i.e. $73 \%$ of people are not aware of drug take back programme and safe drug disposal system.

- Initially, the data shows that more than one-fourth of community is following most improper method of disposing drugs i.e. burning. $28 \%$ of people are using burning method for the disposal of unused, expired or unwanted drugs along with the other solid wastes. The burning of a drug molecule induces several chemical reactions mainly ionization, combustion etc. which will cause the release of toxic gases to the environment.

- For eg: Carbon monoxide -It is a toxic gas released from the incomplete combustion of carbon containing substances and as we know most of drugs chemically made up of carbon as backbone. It not only creates smog but also causes several lung diseases.

- The other toxic substances which will release from the burning of drugs are toxic metals, such as lead and mercury, especially their compounds, volatile organic compounds (VOCs), Chlorofluorocarbons (CFCs), Ammonia $\left(\mathrm{NH}_{3}\right)$ and free radicals of hetero atoms such as oxygen, sulfur and nitrogen etc. These substances not only cause air pollution, but also cause toxicity. Inhalation of toxic gases released by the burning of medications will cause serious health problems such as cancer, cardiovascular diseases, lung diseases, neurological disorders and even death.

- The data shows that $40.18 \%$ of people are throwing the unused or expired medicines into waste basket. If drugs are thrown as such into dustbin there is a chance of accidental poisoning especially in case of children and pets as the drugs have good external appearance for the better patient compliance. Not only this, drugs which are thrown like this into dustbin will end up with either reaching landfills where there is a chance of contamination of water sources and other natural resources or burnt into toxic substances which will cause serious health hazards.

- This major data (40.18\%) suggests that, in India throwing the drugs into dustbin is most common practice and it may be due to lack of knowledge regarding consequences of improper drug disposal.

- From the data, it is come to know that $19.60 \%$ of people are flushing the medications into sink or toilet. In most of the cases people will use this method to dispose liquid preparations. This type of action will not only contaminate the water supplies but also causes serious problems for aquatic life, wild life as well as human beings. Nowadays various water sources are found with trace amount of drugs used for treatment of many diseases. For example, Delhi and Hyderabad crisis as mentioned in introduction part. If a healthy person is frequently exposed to trace amount of drugs through drinking water or from other sources of contaminated water, he will become resistant to those drugs and when he essentially needs that drug for treating a disease it will not be effective anymore i.e. it acts as a vaccine!!.

- One fifth of the population is using this method. So it won't be wrong if I say -'This is the main reason for treatment failure'.

- The data shows that, only $2.80 \%$ of people are returning the unused medicines to the pharmacy. Even though some pharmacies have medicine drop-box system, the utilization of this system was found to be too less through this survey. That means, majority of the people are not aware about the medicine drop-box system and significance of it. Not only this, the data indirectly indicates that majority of the people i.e. $97.2 \%$ of people disposing the medicines themselves. Instead of that they could have return the unused or expired medicine to the pharmacy in order to avoid the consequences of improper drug disposal system.

- The data also shows that, about one-seventh part of these unused, expired medications are antibiotics. Such medications will remain with the patient if he is non-adherent to the medications which are a main reason for the development of drug resistance (Figures 1-5).

- From the data, it was found that about $73 \%$ of people are not aware of drug take back programme or any other safe drug disposal system. However this result may be due to the nonfunctioning of drug take back programme in India. It indicates that there is a need of proper patient education in order to

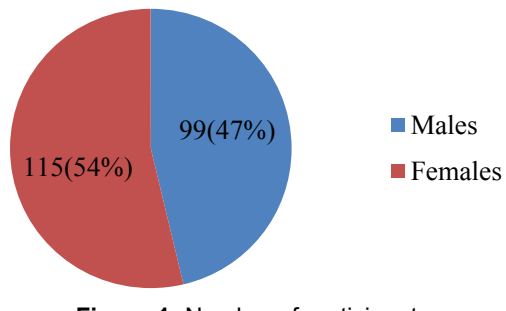

Figure 1: Number of participants. 


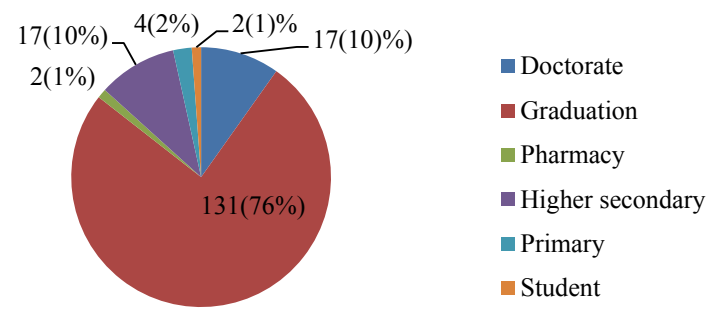

Figure 2: Educational qualifications of participants.

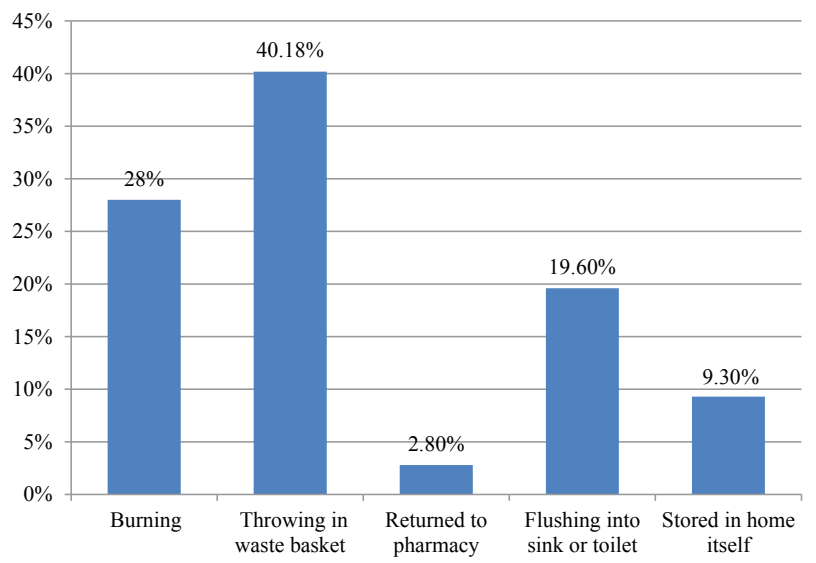

Figure 3: Method of disposal of drugs by people.

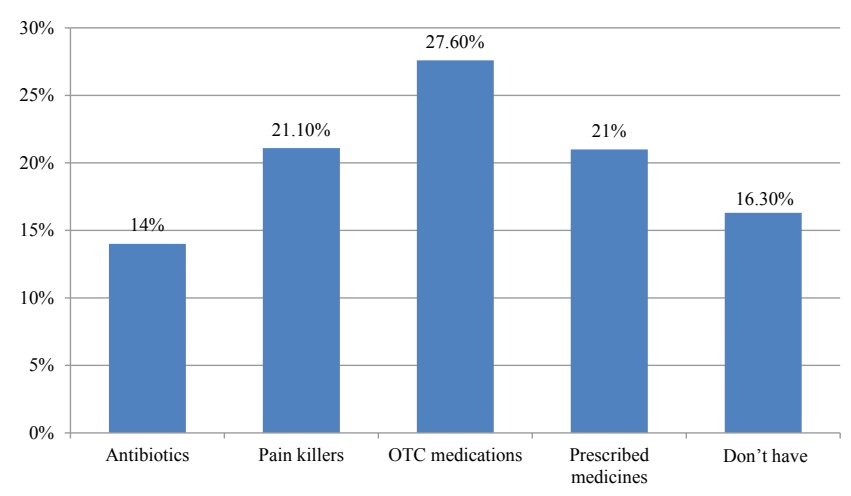

Figure 4: Types of drugs remaining as unused, expired and unwanted medicines with the people.

create awareness regarding disposal of unused or expired medicine safely. It also shows that drug take back programme must develop legislatively to function efficiently.

If any drug take back system is not available then FDA suggest these steps to avoid accidental poisoning. These are as follows:

i. Remove the drugs from their original containers and mix them with something undesirable, such as used coffee grounds, dirt, or cat litter. This makes the medicine less appealing to children and pets and unrecognizable to someone who might intentionally go through the trash looking for drugs.

ii. Put the mixture in something you can close (a re-sealable zipper storage bag, empty can, or other container) to prevent the drug from leaking or spilling out.

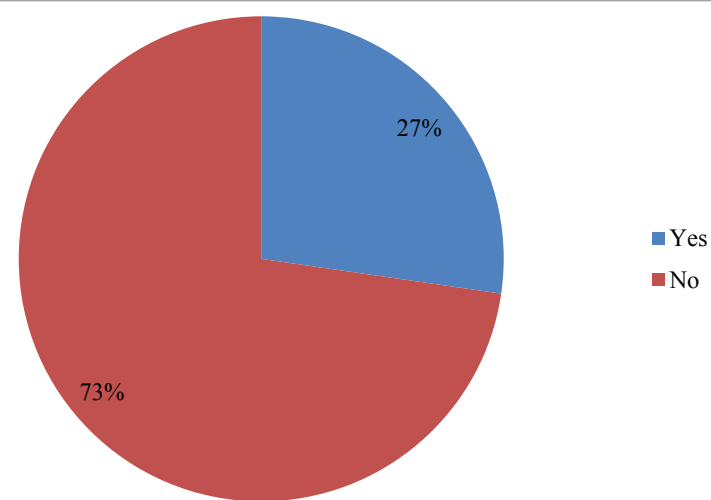

Figure 5: People who aware of drug take back programme or safe drug disposal system.

iii. Throw the container in the garbage.

Scratch out all your personal information on the empty medicine packaging to protect your identity and privacy. Throw the packaging away.

\section{Discussion}

It is a common practice in India that whenever a strip of tablet is seen which may be either unused or expired and if it is not needed any more, the person/citizen will just throw it into the dustbin or waste basket or may burn it along with other waste and if it is a liquid preparation, they will flush it off into sink or toilet by thinking that he is cleaning his home from an unwanted waste. It is absolutely wrong. The actual fact is that, he is not cleaning his home instead polluting the whole environment. These types of dangerous practices are most common in almost every household of India.

As I already mentioned in introduction part, these improper methods will lead to hazardous effects to nature and life such as accidental poisoning, drug abuse, drug resistance, toxicities and even death.

In India, the population is now crossing 135 crores. If every household starts to dispose the drugs by throwing, burning or flushing, there will be one day, where it will be difficult to treat a single disease due to resistance problem and public health will be in the worsen condition due to frequent exposure to toxic substances. So there should be an efficiently working and proper authorized system or programme to control these activities and to ensure public health and safety.

\section{Drug Take Back Programme}

Before the establishments of drug take back programme in USA, the FDA had received more than 30 reports of accidental exposure to the powerful pain medication i.e. fentanyl [11-16] patches and most of them are children under two years old. Tragically 12 of them were hospitalized and 12 of them were died [15]. Not only this, according to Poison Control Centers of United States, in 2007, approximately 23,783 of 255,732 cases involving inappropriate medication use were associated with accidental exposure due to improper disposal [13]. Another data said that, many Americans abuse prescription drugs than the number of those using cocaine, hallucinogens, heroin, and inhalants combined, according to the 2010 National Survey on Drug Use and Health [14]. To prevent and control these problems USFDA initiated Drug take back programme on September $25^{\text {th }} 2010$ with the co-ordination of Drug Enforcement Administration (DEA). 
Citation: Shwetha N, Jha A (2018) Knowledge and Awareness Regarding Safe Drug Disposal System among General Population of India. J Pharmacovigil 6: 256. doi:10.4172/2329-6887.1000256

Page 4 of 4

The drug take back programme not only aims to provide a safe, convenient and responsible means of disposing of prescription drugs but also educate the general public about the potential for abuse and medication.

They will set a particular day as 'drug-take back day' and on that day, DEA authorized collectors will collect unused, unwanted or expired medications from the public. In past years, they have collected more than 200 pounds of unused medications that would have otherwise disposed improperly. Thus it helps to divert medicines from entering the environment. Unfortunately in India, Drug take back programme are not functional (Even though it is mentioned in Pharmaceuticals Export Promotion Council of India [12]) and other systems such as mail drop and medicine drop box are also not efficient. However provisions for the disposal of unused or expired medications from the pharmaceutical industries are given in Bio Medical Waste Management Rules 2016 [16]. But there is no proper and efficient provision for the disposal of drug from each home and to educate the people regarding safe disposal. Because of these one can't even imagine the condition of environment filled with the artistic work of drug molecules with other pollutants from the past century and how badly it is affecting the public health. So there is a need to take efficient steps legislatively to prevent the nature becoming hell..!!!

\section{Other Approaches}

- Awareness programme should be conducted to create awareness among the general population regarding harmful effects of improper drug disposal system.

- There is a need to educate the people that, a simple and silly mistake (disposing drug improperly) can cause a huge loss (death of lives) and hazardous effects to nature as well as public.

- Patient education plays an important role. It is the role of pharmacist or other health care provider to counsel/educate the patient regarding proper storage, utilization as well as proper disposal of medicines.

The study suggests that, the drug take back programme in India has to be developed legislatively.

\section{Conclusion}

According to this study, majority of the people are disposing the drugs improperly and are not aware about the consequences of improper drug disposal. Not only this, many of them are not aware of drug take back system or any other safe drug disposal methods. So there is a need to create awareness and to develop the drug take back programme and other safe drug disposal system legislatively in India.

\section{References}

1. WHO (1999) Guidelines for Safe Disposal of Unwanted Pharmaceuticals in and after Emergencies.

2. Douglas C, Throckmorton (2016) National Drug Take Back Day: A Great Time to Dispose of Prescription Medications Cluttering Your Cabinets.

3. Aditya S, Singh H (2013) Safe medication disposal: Need to sensitize undergraduate students. IJPLS 4: 1-6.

4. Udupa N, Muragundi P, Nagappa A, Janodia M (2017) Disposal of date expired and unused medicines in india-A conceptual framework.

5. Swaroop HS, Charaborty A, Virupakshaiah A (2015) Knowledge, attitude and practice of medical professionals towards the safe disposal of unused medications in South India. World J Pharm Pharm Sci 4: 1423-1430.

6. Maharana SP, Paul B, Dasgupta A, Garg S (2017) Storage, reuse, and disposal of unused medications: A cross-sectional study among rural households of Singur, West Bengal. Int J Med Public Health 1: p1.

7. Kumar M, Saravanan S (2016) Development of Conceptual Framework fo Household Medicine Disposal Practices in India and its Impact on Environment. WJPPS 5: 2269-2280.

8. Radhakrishna L, Nagarajan P (2015) Education on disposal of medicines - A concept on safe disposal of drugs in curriculum of Indian Education system. WJPS 3: 1592-1597.

9. Davies J, Davies D (2010) Origins and evolution of antibiotic resistance. Microbiol Mol Biol Rev 74: 417-433.

10. https://scroll.in/author/13198

11. Lamba M, Ahammad SZ (2017) Sewage treatment effluents in Delhi: A key contributor of $\beta$-lactam resistant bacteria and genes to the environment. Chemosphere 188:249-256.

12. Disposal of Unused Medicines: What You Should Know (2011) Pharmexcil.

13. Bronstein AC, Spyker DA, Louis RC, Green JL, Rumack BH, et al. (2008) "2007 Annual Report of the American Association of Poison Control Centers' National Poison Data System (NPDS): 25th Annual Report". Clinical Toxicol 46: $927-$ 1057.

14. Fass J (2011) Prescription drug take-back programs. 68. Fort Lauderdale, FL: Am J Health Syst Pharm 34: P404.

15. Voice F (2017) National Drug Take Back Day: A Great Time to Dispose of Prescription Medications Cluttering Your Cabinets | FDA Voice.

16. The Bio-Medical Waste Management (Amendment) Rules (2018) 